\title{
Effect of Telmisartan on Olanzapine Induced Metabolic Syndrome in Ovariectomized Female Sprague-Dawley Rats
}

\author{
Vennam Pavani ${ }^{\mathrm{a}}$, Manonmani Alvin Jose ${ }^{\mathrm{a}, \mathrm{c}}$, Shaik Mohammad ${ }^{\mathrm{b}}$, Duraiswami Sathyamurthy, \\ Balasubramanian Nandha kumar ${ }^{\mathrm{a}}$
}

\begin{abstract}
Background: Menopause increases the prevalance of metabolic syndrome in women. Olanzapine is an atypical antipsychotic drug which is used in the treatment of psychiatric disorders, include schizophrenia and bipolar disorder. But it is associated with serious metabolic side-effects, include weight gain, hypertension, hyperlipidemia, hyperglycemia, glucose intolerance and insulin resistance which results in metabolic syndrome. The prevalence of metabolic syndrome is more in patients with schizophrenia compared to the general population. Telmisartan an antihypertensive agent, is an angiotensin II type-I receptor blocker (ARB) also activates peroxisome proliferator-activated receptor gamma (PPAR $\gamma$ ) and provide beneficial effects for glucose and lipid metabolism. Thus the objective of the present study was to evaluate the effect of telmisartan and additional influence of menopause status on olanzapine induced metabolic syndrome in female Sprague-Dawley rats.
\end{abstract}

Methods: After four weeks of ovariectomy, olanzapine $(5 \mathrm{mg} / \mathrm{kg})$ was administered by oral route for 28 days to induce metabolic syndrome in female Sprague-Dawley rats. Thirty female SparagueDawley rats were randomly divided into five groups as normal control; ovariectomy control (OVX); ovariectomy + olanzapine control (OVX+OLZ); OVX + Telmisartan (5 mg/kg); OVX + OLZ + Telmisartan $(5 \mathrm{mg} / \mathrm{kg})$. After 28 days of treatment, the blood samples were collected and analyzed for blood glucose, plasma insulin, lipid profiles, SGOT, SGPT and body weights of all groups were recorded.

Manuscript accepted for publication June 20, 2012

${ }^{a}$ Department of Pharmacology, Swamy Vivekanandha College of Pharmacy, Elayampalayam, Tiruchengode-637205, Tamilnadu, India

${ }^{\mathrm{b}}$ Department of Pharmacology, KLR College of Pharmacy, Palvancha, Kammam (dt), Andhrapradesh, India

${ }^{\mathrm{c} C}$ Corresponding author: Manonmani Alvin Jose, Department of

Pharmacology, Swamy Vivekanandha College of Pharmacy,

Elayampalayam, Tiruchengode-637205, Tamilnadu, India.

Email: pharmjose73@yahoo.co.in

doi:10.4021/jem100w
Results: OVX control and OVX + OLZ control groups showed significant $(\mathrm{P}<0.01)$ increase in body weight, blood glucose, plasma insulin, total cholesterol, triglycerides, LDL-C, VLDL-C, SGOT, SGPT and significant $(\mathrm{P}<0.01)$ decrease in HDL-C when compared to normal control. While OVX group and OVX + OLZ group treated with telmisartan showed significant decrease in body weight gain $(\mathrm{P}<0.05)$, blood glucose $(\mathrm{P}<0.01)$, plasma insulin $(\mathrm{P}<0.01)$, total cholesterol $(\mathrm{P}<0.01)$, triglycerides $(\mathrm{P}<0.05, \mathrm{P}<0.01)$, LDL$\mathrm{C}(\mathrm{P}<0.01)$, VLDL-C $(\mathrm{P}<0.05, \mathrm{P}<0.01)$, SGOT $(\mathrm{P}<0.05, \mathrm{P}<$ $0.01)$, SGPT $(\mathrm{P}<0.01)$ and significant increase in HDL-C $(\mathrm{P}<$ 0.01 ) when compared to OVX control and OVX + OLZ control group. The results of histopathological studies provide strong support to our results.

Conclusions: Telmisartan attenuate the development of metabolic syndrome induced by olanzapine in ovariectomized female Sprague-Dawley rats.

Keywords: Menopause; Ovariectomy; Metabolic syndrome; Insulin resistance; Hyperlipidemia; Hyperglycemia; Peroxisome proliferator-activated receptor gamma (PPAR $\gamma)$

\section{Introduction}

Metabolic syndrome is a combination of metabolic risk factors that include abdominal obesity, atherogenic dyslipidemia, hypertension, and insulin resistance which increases the risk of cardiovascular disease and type-2 diabetes [1]. It is also called as syndrome $\mathrm{X}$, dysmetabolic syndrome, cardiometabolic syndrome, insulin resistance syndrome, Reaven's syndrome, the deadly quartet, GHO (glucose intolerance, hypertension, obesity) syndrome [2, 3]. Metabolic syndrome is a rising clinical challenge and is classified as a disease entity by the Center for Disease Control (CDC) and has an international classification of disease code of 277.7 [4]. Metabolic syndrome is the one of the most common chronic diseases worldwide and the fourth or fifth leading cause of death in the developed world [5]. It is estimated that around $20-25$ percent of the world's adult population have the metabolic syndrome and they are twice as likely to 
Table 1. Effect of Telmisartan on Body Weight

\begin{tabular}{|c|c|c|c|c|}
\hline \multirow{2}{*}{ Group } & \multirow{2}{*}{ Treatment } & \multicolumn{3}{|c|}{ Body weight (g) } \\
\hline & & Initial & Final & Weight gain \\
\hline A & Normal Control & $211.67 \pm 6.009$ & $223.50 \pm 6.087$ & $11.833 \pm 0.910$ \\
\hline B & OVX Control & $213.33 \pm 9.545$ & $233.83 \pm 10.879$ & $20.500 \pm 2.432^{* *}$ \\
\hline $\mathrm{C}$ & OVX + OLZ Control & $220.00 \pm 11.255$ & $242.16 \pm 11.677$ & $22.167 \pm 2.242^{* *}$ \\
\hline $\mathrm{D}$ & OVX + TEL & $225.00 \pm 9.574$ & $211.50 \pm 10.052$ & $13.500 \pm 1.544^{\S}$ \\
\hline $\mathrm{E}$ & $\mathrm{OVX}+\mathrm{OLZ}+\mathrm{TEL}$ & $220.00 \pm 7.303$ & $205.33 \pm 7.401$ & $14.667 \pm 1.453^{\#}$ \\
\hline
\end{tabular}

$\mathrm{n}=6$; the values are expressed as Mean $\pm \mathrm{SEM}$; ${ }^{* *} \mathrm{P}<0.01$ when compared to normal control, ${ }^{\$} \mathrm{P}<0.05$ when compared to OVX and "P $<0.05$ when compared to OVX + OLZ (One way ANOVA followed by Dunnett's multiple comparison test). OVX: ovariectomy control, OVX + OLZ: ovariectomy + olanzapine control, OVX + TEL: ovariectomy group treated with telmisartan, OVX + OLZ + TEL: ovariectomy + olanzapine group treated with telmisartan.

die from it, and three times as likely to have a heart attack or stroke compared with people without the syndrome. In addition, people with metabolic syndrome have a fivefold greater risk of developing type-2 diabetes [5]. The prevalence of the metabolic syndrome increases with age, but there are ethnic and national differences [6].

Menopause heralds a decline in circulating estrogen levels, which may increase cardiovascular risk through the effects on adiposity, lipid metabolism, and prothrombotic state. It has been suggested that all these modifications may result in a menopausal metabolic syndrome, as many of the risk factors are more prevalent in postmenopausal women [1]. Menopause increases the prevalence of metabolic syn- drome in women. Menopause condition is induced experimentally in rats by ovariectomy [1]. These ovariectomy (OVX) induced metabolic changes might be caused directly by estrogen deficiency and may occur partly as secondary effects of obesity arising due to the orexigenic effects of estrogen deficiency [7].

Olanzapine is the one of the most commonly prescribed atypical antipsychotic drugs worldwide which is used in the treatment of chronic psychotic disorders, include schizophrenia and bipolar disorder [8]. It acts by antagonizing the actions of dopamine $\left(\mathrm{D}_{2}\right)$ receptors and serotonin $(5-\mathrm{HT} 2 \mathrm{~A} / 2 \mathrm{C})$ receptors. By blocking these dopamine $\left(\mathrm{D}_{2}\right)$ and serotonin $(5-\mathrm{HT} 2 \mathrm{~A} / 2 \mathrm{C})$ receptors olanzapine reduces extrapyramidal

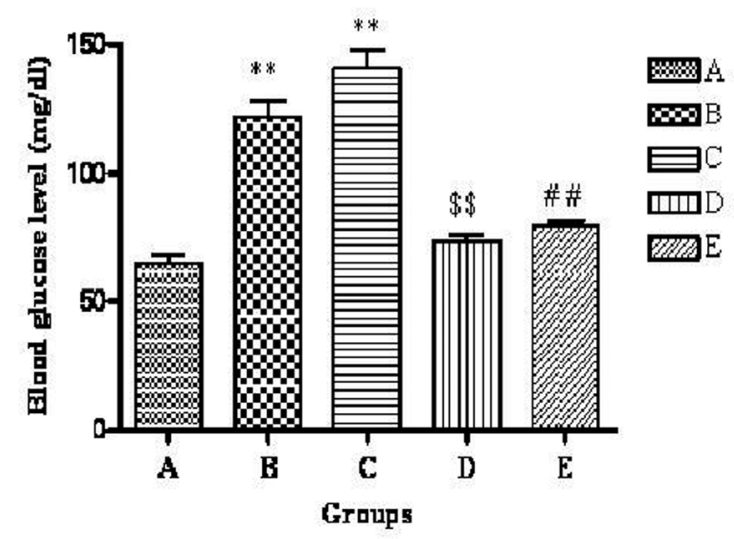

Figure 1. Effect of telmisartan on blood glucose level. $n=6$; The values are expressed as mean \pm SEM; ${ }^{* *} \mathrm{P}<0.01$ when compared to normal control, ${ }^{\$ \$} \mathrm{P}<0.01$ when compared to OVX and ${ }^{\# P} \mathrm{P}<$ 0.01 when compared to OVX + OLZ (One way ANOVA followed by Dunnett's multiple comparison test). Group A: normal control, Group B: ovariectomy control, Group C: ovariectomy + olanzapine control, Group D: ovariectomy group treated with telmisartan, Group E: ovariectomy + olanzapine group treated with telmisartan. 


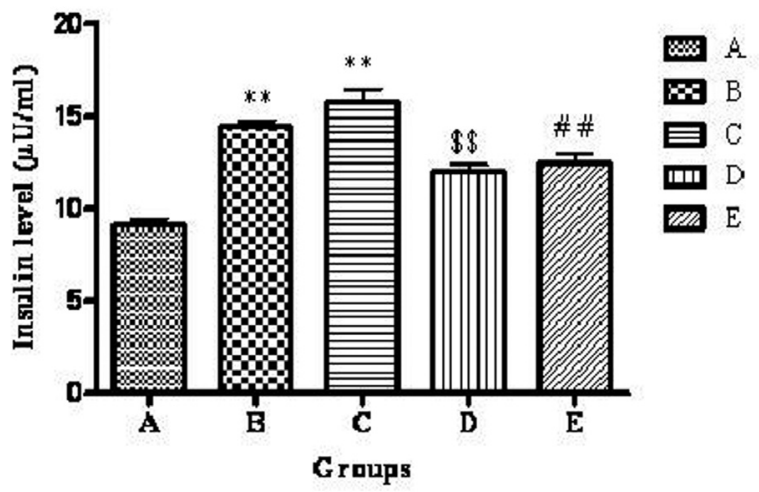

Figure 2. Effect of telmisartan on plasma insulin level. $n=6$; The values are expressed as mean \pm SEM; ${ }^{*} \mathrm{P}<0.01$ when compared to normal control, ${ }^{\$ \$} \mathrm{P}<0.01$ when compared to OVX and ${ }^{\#} \mathrm{P}<$ 0.01 when compared to OVX + OLZ (One way ANOVA followed by Dunnett's multiple comparison test). Group A: normal control, Group B: ovariectomy control, Group C: ovariectomy + olanzapine control, Group D: ovariectomy group treated with telmisartan, Group E: ovariectomy + olanzapine group treated with telmisartan.

side effects [9]. There are several evidences indicating that atypical antipsychotic drugs have serious adverse effects. These primarily include metabolic side-effects, which substantially increase the risk of developing cardio-metabolic disorders such as type-2 diabetes mellitus (DM) and cardiovascular diseases. In the clinical setting, the identifying characteristics of antipsychotic drug-induced metabolic disorders are weight gain, hypertension, hyperlipidemia, hyperglycemia, glucose intolerance and insulin resistance [8]. Olanzapine and clozapine are more prone to induce these metabolic side-effects than other atypical antipsychotic drugs [10]. Many reports have found a higher prevalence of metabolic syndrome in patients with schizophrenia compared to the general population [11].

Telmisartan an antihypertensive agent, is an angiotensin II type-I receptor blocker (ARB) which is highly selective for the angiotensin II type $1\left(\mathrm{AT}_{1}\right)$ receptor. It acts by blocking the renin-angiotensin-aldosterone system (RAAS) by inhibiting the actions of angiotensin II at the angiotensin II type $1\left(\mathrm{AT}_{1}\right)$ receptor [12]. For all angiotensin receptor blockers (ARBs), telmisartan has demonstrated the ability not only to inhibit angiotensin II type 1 receptor but also to activate peroxisome proliferator-activated receptor gamma (PPAR $\gamma$ ) and to provide beneficial effects for glucose and lipid metabolism. Telmisartan acts as a selective PPAR $\gamma$ modulator and modifies the expression of numerous metabolism-related genes and plays a major role in glucose metabolism [13]. Because of its greater PPAR $\gamma$-agonist activity, telmisartan has been reported to be associated with improvements in insulin resistance and glucose metabolism compared with other angiotensin receptor blockers [14].

Thus in the present study the effect of telmisartan and additional influence of menopause status on olanzapine induced metabolic syndrome was investigated in ovariecto- mized female Sprague-Dawley rats.

\section{Materials and Methods}

\begin{abstract}
Animals
Female Sprague-Dawley rats of 6 - 8 weeks old, weighing 200 - 220 g were obtained from Swamy Vivekanandha College of Pharmacy, animal house were used for the study. Throughout the acclimatization and experimental period, the animals were housed in autoclavable polypropylene cages under standard laboratory conditions at $21 \pm 2{ }^{\circ} \mathrm{C}$ and 50 $60 \%$ relative humidity with a photo period of 12 -h light/12-h dark cycle. The animals were provided with standard pellet diet and water ad libitum. Study protocol was approved by Institutional Animal Ethical Committee (IAEC), Swamy Vivekanandha College of Pharmacy (Proposal No: SVCP/ IAEC/M.Pharm/07/Sep/2011) and experiments were conducted in accordance with guidelines set by the CPCSEA (Committee for the Purpose of Control and Supervision of Experiment on Animals), India.
\end{abstract}

\section{Drugs and Chemicals}

Gift samples of each drug were obtained from various pharmaceutical companies along with their analytical data, Olanzapine (Sigma chemicals and drugs, Hyderabad) Telmisartan (Ranbaxy Laboratories Limited, Mumbai). The other chemicals were purchased from various companies Carboxy methyl cellulose (Loba Chemicals Pvt. Ltd., Mumbai), Formaldehyde (Nice Chemicals Pvt. Ltd., Kochi), Sodium phosphate monobasic (Loba Chemicals Pvt. Ltd., Mumbai), Sodium phosphate dibasic (Loba Chemicals Pvt. Ltd., Mum- 
bai), Neosporin (Glaxo Smithkline pharmaceutical Ltd., Bangalore), Povidine-iodine solution (Cipla Ltd., Mumbai) and used for the study.

\section{Experimental design}

The thirty female Sprague-Dawley rats were randomly divided into five groups. Group A: Normal control (1\% w/v CMC in water), Group B: Disease control (ovariectomy + $1 \% \mathrm{w} / \mathrm{v} \mathrm{CMC}$ in water), Group C: Disease control (ovariectomy + olanzapine $5 \mathrm{mg} / \mathrm{kg} /$ day in $1 \% \mathrm{w} / \mathrm{v}$ CMC), Group D: Disease $(\mathrm{OVX})+$ Telmisartan $(5 \mathrm{mg} / \mathrm{kg} /$ day in $1 \% \mathrm{w} / \mathrm{v}$ CMC), Group E: Disease (OVX + Olanzapine) + Telmisar$\tan (5 \mathrm{mg} / \mathrm{kg} /$ day in $1 \% \mathrm{w} / \mathrm{v} \mathrm{CMC})$. Each group containing six animals $(n=6)$. Ovariectomy $(\mathrm{OVX})$ was performed for all 4 groups except normal control group. Animals were allowed for one month to recover. After the recovery, drug treatment was given to all groups. The experimental menopause was induced by ovariectomy in rats. Ensured that this menopause period itself induces metabolic syndrome in rats. Also metabolic syndrome in rats was induced by administering olanzapine $5 \mathrm{mg} / \mathrm{kg}$, once a day by oral route at 10.00 am to the respective groups using an intragastric tube for 28 days and treatment with telmisartan was given by oral route, once a day, at $3.00 \mathrm{pm}$ to respective groups for the period of 28 days.

Initial and final body weights of all groups were recorded. At the end of the experimental period, the animals were fasted for overnight and blood was collected in EDTA tubes by cardiac puncture. Plasma was analyzed for glucose, insulin, TC, TGs, HDL-C, LDL-C, VLDL-C, SGOT, and SGPT by enzymatic kits using semi-auto analyzer. Then animals of all groups were sacrificed by decapitation and immediately liver, kidneys were dissected, washed in ice-cold saline to remove the blood, then weights of liver and kidney were recorded. The averages of all parameters were computed.

\section{Blood and plasma biochemical analysis}

Blood glucose was determined using Trinder's glucose oxidase and proxidase method [15] while plasma insulin was determined by ELISA method of using UBI MAGWELL Diagnostic kit (United Biotech Inc., California, USA) [16]. Plasma total cholesterol was determined by cholesterol oxidase-perixodase (CHOD - POD) method of using Accurex Cholestrol kit (Accurex Biomedical Pvt. Ltd., Thane, India) [17] and triglycerides were determined by glucose oxidaseperoxidase (GOD - POD) method with N-Ethyl-N Sulfopropyl-N-Anisidine (ESPAS) was used by using Accurex Triglyceride kit (Accurex Biomedical Pvt. Ltd., Thane) [18] respectively. HDL-cholesterol was then determined using the aforementioned cholesterol kit. LDL-cholesterol was calculated using the levels of total cholesterol, triglycerides, HDL cholesterol obtained using the Friedewald formula [19]. 


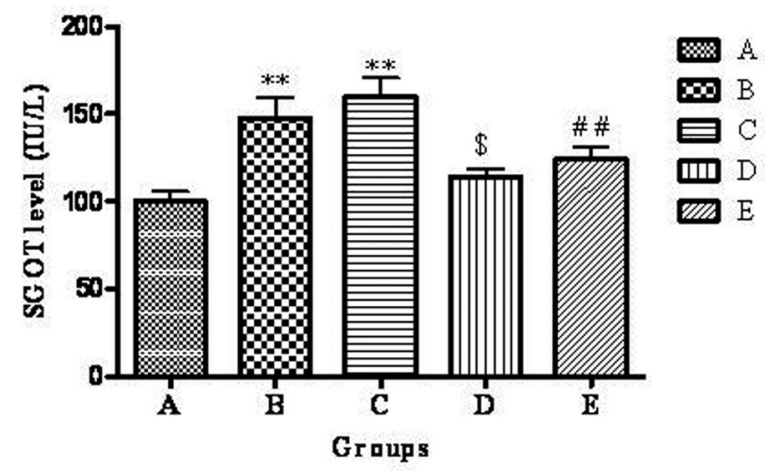

Figure 3. Effect of telmisartan on SGOT level. $n=6$; The values are expressed as mean $\pm \mathrm{SEM}$; ${ }^{* *} \mathrm{P}<0.01$ when compared to normal control, ${ }^{\circledR} \mathrm{P}<0.05$ when compared to OVX and ${ }^{\#} \mathrm{P}<0.01$ when compared to OVX + OLZ (One way ANOVA followed by Dunnett's multiple comparison test). Group A: normal control, Group B: ovariectomy control, Group C: ovariectomy + olanzapine control, Group D: ovariectomy group treated with telmisartan, Group E: ovariectomy + olanzapine group treated with telmisartan.

\section{Histopathological studies}

After 28 days, during necropsy, liver was resected, fixed in $10 \%$ buffered formalin $(37-40 \%$ Formaldehyde $(100 \mathrm{~mL})$, Sodium phosphate monobasic $(4 \mathrm{~g})$, Sodium phosphate dibasic $(6.5 \mathrm{~g})$ and Distilled water $(900 \mathrm{~mL}))$, and were further processed for histopathological examination.

\section{Statistical analysis}

The values are expressed as mean \pm SEM. One way analysis of variance (ANOVA) followed by Dunnett's multiple comparison test was used to analyse the effect of telmisartan when compared to control and disease control groups, by using Graph Pad Instat software, version 3.01; P $<0.05$ was considered as significant.

\section{Results}

\section{Effect of telmisartan on body weight}

The body weight of rats in group $\mathrm{B}$ and $\mathrm{C}$ showed significant $(\mathrm{P}<0.01)$ increase when compared to group A. Group D showed significant $(\mathrm{P}<0.05)$ decrease in body weight when compared to group B. Group E showed significant $(\mathrm{P}<0.05)$ decrease in body weight when compared to group $\mathrm{C}$ (Table 1).

\section{Effect of telmisartan on blood glucose level}

The mean blood glucose levels of groups A, B, C, D and E were $64.55 \pm 3.618,121.83 \pm 6.35,140.50 \pm 7.14,73.03 \pm$ $2.66,79.48 \pm 1.77 \mathrm{mg} / \mathrm{dL}$ respectively. Group B and C had

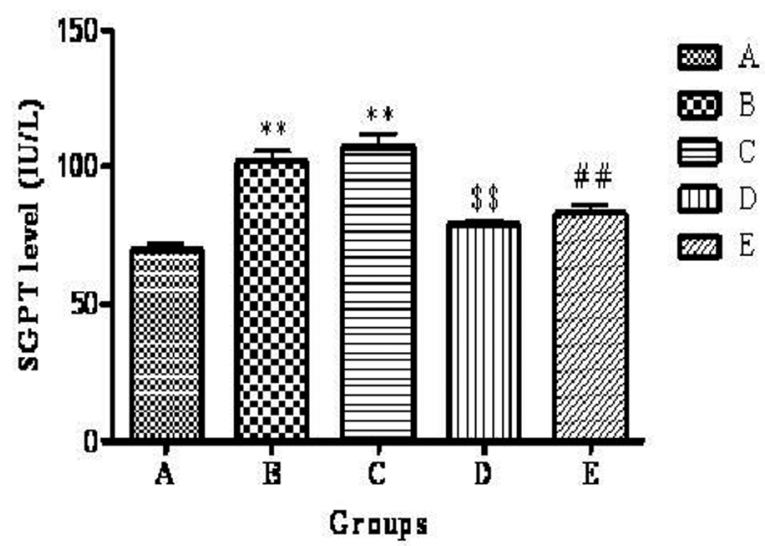

Figure 4. Effect of telmisartan on SGPT level. $n=6$; The values are expressed as mean $\pm \mathrm{SEM}$; ${ }^{* *} \mathrm{P}<0.01$ when compared to normal control, ${ }^{\$ \$} \mathrm{P}<0.01$ when compared to OVX and ${ }^{\# \#} \mathrm{P}<0.01$ when compared to OVX + OLZ (One way ANOVA followed by Dunnett's multiple comparison test). Group A: normal control, Group B: ovariectomy control, Group C: ovariectomy + olanzapine control, Group D: ovariectomy group treated with telmisartan, Group E: ovariectomy + olanzapine group treated with telmisartan. 
Table 3. Effect of Telmisartan on Liver and Kidneys Weight

\begin{tabular}{llll}
\hline Group & Treatment & Liver weight & Kidney weight \\
\hline A & Normal Control & $2.210 \pm 0.06$ & $0.680 \pm 0.035$ \\
B & OVX Control & $3.267 \pm 0.123^{* *}$ & $0.872 \pm 0.047^{* *}$ \\
C & OVX + OLZ Control & $3.350 \pm 0.161^{* *}$ & $0.944 \pm 0.045^{* *}$ \\
D & OVX + TEL & $2.382 \pm 0.110^{\$ s}$ & $0.723 \pm 0.025^{\$}$ \\
E & OVX + OLZ + TEL & $2.367 \pm 0.096^{\# \#}$ & $0.797 \pm 0.032^{\#}$ \\
\hline
\end{tabular}

$\mathrm{n}=6$; The values are expressed as mean $\pm \mathrm{SEM} ;{ }^{* *} \mathrm{P}<0.01$ when compared to normal control group, ${ }^{\$ \$ P}<0.01,{ }^{\$ P}<0.05$, when compared to OVX and ${ }^{\# P} \mathrm{P}<0.01$, ${ }^{\#} \mathrm{P}<0.05$ when compared to OVX $+\mathrm{OLZ}$ (One way ANOVA followed by Dunnet t's multiple comparison test).

a significant $(\mathrm{P}<0.01)$ increase in blood glucose level compared to group A. Group D showed significant $(\mathrm{P}<0.01)$ decrease in blood glucose level when compared to group B. Group E showed significant $(\mathrm{P}<0.01)$ decrease in blood glucose level when compared to group C (Fig. 1).

\section{Effect of telmisartan on plasma insulin level}

The mean plasma insulin level of groups A, B, C, D and E were $9.10 \pm 0.35,14.46 \pm 0.32,15.78 \pm 0.677,12.01 \pm 0.35$, $12.43 \pm 0.55 \mu \mathrm{U} / \mathrm{mL}$ respectively. The highest plasma insulin level was observed in group C. There was a significant $(\mathrm{P}<0.01)$ increase in group $\mathrm{B}$ and $\mathrm{C}$ compared to group $\mathrm{A}$. Group D showed significant $(\mathrm{P}<0.01)$ decrease in plasma insulin level when compared to group B. Group E showed significant $(\mathrm{P}<0.01)$ decrease in plasma insulin level when compared to group C (Fig. 2).

\section{Effect of telmisartan on lipid profile}

The cholesterol level of rats in group $\mathrm{B}$ and $\mathrm{C}$ showed significant $(\mathrm{P}<0.01)$ increase when compared to group A. Group $\mathrm{D}$ showed significant $(\mathrm{P}<0.01)$ decrease in cholesterol level when compared to group B. Group E showed significant $(\mathrm{P}<$ 0.01) decrease in cholesterol level when compared to group C.

The triglyceride level of rats in group $\mathrm{B}$ and $\mathrm{C}$ showed significant $(\mathrm{P}<0.01)$ increase when compared to group A. Group D showed significant $(\mathrm{P}<0.05)$ decrease in triglyceride level when compared to group B. Group E showed significant $(\mathrm{P}<0.01)$ decrease in triglyceride level when compared to group $\mathrm{C}$.

The HDL-C level of rats in group B and C showed significant $(\mathrm{P}<0.01)$ decrease when compared to group $\mathrm{A}$. Group D showed significant $(\mathrm{P}<0.01)$ increase in HDL-C level when compared to group B. Group E showed signifi- cant $(\mathrm{P}<0.01)$ increase in HDL-C level when compared to group $\mathrm{C}$.

The LDL-C level of rats in group B and C showed significant $(\mathrm{P}<0.01)$ increase when compared to group A. Group D showed significant $(\mathrm{P}<0.01)$ decrease in LDL-C when compared to group B. Group E showed significant $(\mathrm{P}<0.01)$ decrease in LDL-C level when compared to group $\mathrm{C}$.

The VLDL-C level of rats in group $\mathrm{B}$ and $\mathrm{C}$ showed significant $(\mathrm{P}<0.01)$ increase when compared to group A. Group D showed significant $(\mathrm{P}<0.05)$ decrease in triglyceride level when compared to group B. Group E showed significant $(\mathrm{P}<0.01)$ decrease in triglyceride level when compared to group $\mathrm{C}$ (Table 2).

\section{Effect of telmisartan on SGOT level}

The mean plasma SGOT level of groups A, B, C, D and E were $99.95 \pm 5.88,147.93 \pm 11.73,160.31 \pm 10.32,114.00 \pm$ $5.086,124.20 \pm 6.814 \mathrm{IU} / \mathrm{L}$ respectively. The highest plasma SGOT level was observed in group $\mathrm{C}$. There was a significant $(\mathrm{P}<0.01)$ increase in group $\mathrm{B}$ and $\mathrm{C}$ compared to group A. Group D showed significant $(\mathrm{P}<0.05)$ decrease in plasma SGOT level when compared to group B. Group E showed significant $(\mathrm{P}<0.01)$ decrease in plasma SGOT level when compared to group $\mathrm{C}$ (Fig. 3).

\section{Effect of telmisartan on SGPT level}

The mean plasma SGPT level of groups A, B, C, D and E were $69.60 \pm 2.22,101.56 \pm 3.99,107.25 \pm 4.84,78.86 \pm$ $1.71,83.10 \pm 2.79 \mathrm{IU} / \mathrm{L}$ respectively. The highest plasma SGPT level was observed in group $C$. There was a significant $(\mathrm{P}<0.01)$ increase in group $\mathrm{B}$ and $\mathrm{C}$ compared to group A. Group D showed significant $(\mathrm{P}<0.01)$ decrease in plasma SGPT level when compared to group B. Group E showed significant $(\mathrm{P}<0.01)$ decrease in plasma SGPT level when 
compared to group C (Fig. 4).

\section{Effect of telmisartan on liver and kidneys weight}

The liver weight of rats in Group B and C showed significant $(\mathrm{P}<0.01)$ increase when compared to group A. Group D showed significant $(\mathrm{P}<0.01)$ decrease in liver weight when compared to group B. Group E showed significant $(\mathrm{P}<0.01)$ decrease in liver weight when compared to group $\mathrm{C}$.

The kidneys weight of rats in Group B and C showed significant $(\mathrm{P}<0.01)$ increase when compared to group A. Group D showed significant $(\mathrm{P}<0.05)$ decrease in kidneys weight when compared to group $\mathrm{B}$. Group $\mathrm{E}$ showed significant $(\mathrm{P}<0.05)$ decrease in kidneys weight when compared to group $\mathrm{C}$ (Table 3 ).

\section{Histopathology of liver}

After 28 days of treatment, the normal control group showed liver depicting normal radiating cords of hepatocytes and central vein. Ovariectomy control group showed severe diffuse fatty changes (large vacuoles) within the hepatocytes of liver and ovariectomy + olanzapine control group showed severe fatty changes (large vacuoles) within almost all hepatocytes of liver. Ovariectomized rats treated with telmisartan showed mild fatty changes (small vacuoles) within the few hepatocytes of liver. Ovariectomy + olanzapine group treated with telmisartan showed mild fatty change (small vacuoles) within the stray hepatocytes of liver.

\section{Discussion}

Metabolic syndrome is a common precursor of cardiovascular disease and type- 2 diabetes that is characterized by the clustering of insulin resistance/hyperinsulinemia, dyslipidemia and increased blood pressure [20]. It affects one in five people in the United States and prevalence increases with age [21].

Menopause may increase the risk of cardiovascular disease through the effects on adiposity, lipid metabolism, prothrombotic state because of decreased levels of estrogen which may result in menopausal metabolic syndrome. Menopause condition is induced experimentally by ovariectomy in animals [1]. Ovariectomy-induced estrogen deficiency results in significantly increased weight gain and increased in risk factors for the metabolic syndrome, such as hyperglycemia, hyperinsulinemia, and insulin resistance. Ovariectomized rats showed estrogen depletion, which triggered the metabolic syndrome and some renal metabolic abnormalities [22].

Olanzapine is an atypical antipsychotic drug which is used in the treatment of psychiatric disorders but it is associated with numerous adverse side-effects including meta- bolic syndrome. Metabolic side-effects such as weight gain, adiposity, dyslipidaemia, glucose dysregulation and insulin resistance are particularly problematic, and leading to further social and medical consequences including obesity, cardiovascular disease, type-2 diabetes [23]. In this present study metabolic syndrome is induced by olanzapine and is potentiated by ovariectomy in female Sprague-Dawley rats.

Telmisartan an antihypertensive agent, is an angiotensin II type-I receptor blocker (ARB) and also activates PPAR $\gamma$, which affects the expression of key enzymes that mediate the beneficial effects of glucose and lipid metabolism. Telmisartan may improve glucose and lipid metabolism with the reduction in visceral fat mass in patients with type-2 diabetes and metabolic syndrome [13]. It has been reported to improve insulin sensitivity (IS) in insulin-resistant subjects [24] and also reduces glucose, insulin, and triglyceride levels [25]. Thus, telmisartan may be used not only in the treatment of hypertension but also in the prevention of metabolic syndrome [13].

The objective of our study was to evaluate the effects of telmisartan on olanzapine induced metabolic syndrome and to evaluate the additional influence of a menopause status in female Sprague-Dawley rats.

In the present study, ovariectomized rats showed significant increase in weight gain, blood glucose, insulin, total cholesterol, triglycerides, LDL-C, VLDL-C, SGOT, SGPT and decrase in HDL-C which are consistent with the reports of Choi et al (2009), who reported that the ovariectomy-induced estrogen deficiency results in significantly increased weight gain, glucose concentration, insulin, cholesterol, triglyceride levels and increases in risk factors for the metabolic syndrome, such as hyperglycemia, hyperinsulinemia, and insulin resistance [22]. Bitto et al (2009) reported that the HDL-C level was decreased and LDL-C level was increased significantly in ovariectomised (OVX) rats [1]. Hamed et al (2010) reported that the VLDL-C level was increased in ovariectomised female albino rats [26]. Choi et al (2009) reported that ovarian hormones exert significant control over insulin resistance and cellular lipid homeostasis in metabolically active tissues, such as the liver and skeletal muscle [22].

Our results also confirmed that olanzpine administration to ovariectomized rats showed significant increase in weight gain, blood glucose, insulin, total cholesterol, triglycerides, LDL-C, VLDL-C, SGOT, SGPT and decrase in HDL-C which are consistent with the reports of Coccurello et al (2008) who reported that olanzapine 3 and $6 \mathrm{mg} / \mathrm{kg}$ significantly increases the body weight, glucose, insulin, cholesterol, triglyceride level during the last 10 days of treatment in mice [27]. Del Valle et al (2006) reported that olanzapine decreases HDL-C and increases LDL-C level in patients with schizophrenia [28]. Sofic et al (2007) reported that olanzapine increases plasma VLDL-C level in patients with psychosis [29]. Sengupta et al (2006) reported that SGOT, SGPT 
levels were increased in Wistar albino rats when treated with olanzapine [30].

Telmisartan did not restore serum estradiol to the normal levels, it did attenuate ovariectomy and olanzapine induced insulin resistance. Thus, the preventative effect of telmisartan against insulin resistance may be the result of the improving glucose and lipid metabolism through modulation of PPAR $\gamma$.

Telmisartan treatment to ovariectomized rats and ovariectomy + olanzapine group attenuates the development of metabolic syndrome, by reducing the body weight and decreasing the levels of glucose, insulin, total cholesterol, triglycerides, LDL-C, VLDL-C, SGOT, SGPT and increased levels of HDL-C in blood in the present study. Our results are consistent with the reports of Kakuma et al (2010), who reported that telmisartan reduces the body weight, triglyceride, and improve HDL-C, glucose and lipid metabolism with the reduction in visceral fat mass in patients with type-2 diabetes and metabolic syndrome [13]. Makitha et al (2008) reported that telmisartan reduces cholesterol level in hypertensive patients with glucose intolerance [31]. Rizos et al (2008), who reported that telmisartan decreases LDL-C level in patients with the metabolic syndrome [14]. Goyal et al (2008), who reported that telmisartan reduces VLDL-C level in diabetic rats [32]. Clemenz et al (2008) reported that telmisartan restores impaired SGOT, SGPT levels to normal level [33].

The fat accumulation in the liver is associated with insulin resistance, which can progress to diabetes [34]. Our results showed that ovariectomy and olanzapine induced an insulin resistance and a tendency to an increase in hepatic fat deposit. However, telmisartan improved the ovariectomy and olanzapine induced fatty changes by improving glucose and lipid metabolism.

Strength of our study was the effects of telmisartan on metabolic syndrome were done only in normal rats till now. But there was no such studies done by using telmisartan on olanzapine induced metabolic syndrome in ovariectomized rats. Our study is the first report to study the effect of telmisartan on olanzapine induced metabolic syndrome in ovariectomized female Sprague-Dawley rats.

The limitation of our study includes that we might have evaluated the blood pressure because blood pressure is the one of the important component for the diagnosis of metabolic syndrome [35]. Patil et al (2006) reported that chronic treatment with olanzapine (1 and $2 \mathrm{mg} / \mathrm{kg}$ ) elevates systolic blood pressure in normal rats [10]. Derosa et al (2004) reported that telmisartan reduces blood pressure in patients with type-2 diabetes mellitus and mild hypertension [12].

Telmisartan may also protect occurrence of nephropathy in diabetes patients so we suggested that further studies may be carried out in this direction.

This preliminary investigation revealed that telmisartan decreased the levels of glucose, insulin, total cholesterol, triglycerides, LDL-C, VLDL-C, SGOT, SGPT and also showed significant increase in the reduced levels of HDL-C by telmisartan on olanzapine induced metabolic syndrome in ovariectomized female Sprague-Dawley rats. Thus telmisartan may attenuate metabolic syndrome and modulate glucose and lipid metabolism by activating peroxisome proliferatoractivated receptor gamma (PPAR $\gamma)$.

\section{Conclusion}

Telmisartan attenuate the development of metabolic syndrome induced by olanzapine in ovariectomized female Sprague-Dawley rats. Therefore our study recommended that telmisartan may be used for the treatment of metabolic syndrome in schizophrenic menopausal women treated with olanzapine after proving its effects in humans with well controlled clinical trials.

\section{Conflict of Interest}

There is no potential conflict of interest relevant to this article.

\section{References}

1. Bitto A, Altavilla D, Bonaiuto A, Polito F, Minutoli L, Di Stefano V, Giuliani D, et al. Effects of aglycone genistein in a rat experimental model of postmenopausal metabolic syndrome. J Endocrinol. 2009;200(3):367376.

2. Meyer JM, Davis VG, Goff DC, McEvoy JP, Nasrallah HA, Davis SM, Rosenheck RA, et al. Change in metabolic syndrome parameters with antipsychotic treatment in the CATIE Schizophrenia Trial: prospective data from phase 1. Schizophr Res. 2008;101(1-3):273-286.

3. Reaven GM. Banting lecture 1988. Role of insulin resistance in human disease. Diabetes. 1988;37(12):15951607.

4. Babu A, Fogelfeld L. Metabolic syndrome and prediabetes. Dis Mon. 2006;52(2-3):55-144.

5. Alberti KG, Zimmet P, Shaw J. The metabolic syndrome--a new worldwide definition. Lancet. 2005;366(9491):1059-1062.

6. Day C. Metabolic syndrome, or What you will: definitions and epidemiology. Diab Vasc Dis Res. 2007;4(1):32-38.

7. Kim JY, Jo KJ, Kim OS, Kim BJ, Kang DW, Lee KH, Baik HW, et al. Parenteral 17-beta-estradiol decreases fasting blood glucose levels in non-obese mice with short-term ovariectomy. Life Sci. 2010;87(11-12):358366.

8. Boyda HN, Procyshyn RM, Tse L, Wong D, Pang CC, Honer WG, Barr AM. Intermittent treatment with olan- 
zapine causes sensitization of the metabolic side-effects in rats. Neuropharmacology. 2012;62(3):1391-1400.

9. Assie MB, Carilla-Durand E, Bardin L, Maraval M, Aliaga M, Malfetes N, Barbara M, et al. The antipsychotics clozapine and olanzapine increase plasma glucose and corticosterone levels in rats: comparison with aripiprazole, ziprasidone, bifeprunox and F15063. Eur J Pharmacol. 2008;592(1-3):160-166.

10. Patil BM, Kulkarni NM, Unger BS. Elevation of systolic blood pressure in an animal model of olanzapine induced weight gain. Eur J Pharmacol. 2006;551(1-3):112-115.

11. Lee NY, Kim SH, Jung DC, Kim EY, Yu HY, Sung $\mathrm{KH}$, Kang UG, et al. The prevalence of metabolic syndrome in Korean patients with schizophrenia receiving a monotherapy with aripiprazole, olanzapine or risperidone. Prog Neuropsychopharmacol Biol Psychiatry. 2011;35(5):1273-1278.

12. Derosa G, Cicero AF, Bertone G, Piccinni MN, Fogari E, Ciccarelli L, Fogari R. Comparison of the effects of telmisartan and nifedipine gastrointestinal therapeutic system on blood pressure control, glucose metabolism, and the lipid profile in patients with type 2 diabetes mellitus and mild hypertension: a 12-month, randomized, double-blind study. Clin Ther. 2004;26(8):1228-1236.

13. Kakuma T, Gotoh K, Masaki T, Itateyama E, Abe N, Yoshimatsu H. Telmisartan reduced abdominal circumference and body weight with decreasing triglyceride level in patients with type 2 diabetes and metabolic syndrome. Obes Res and Clin Pract. 2010;4:145-152

14. Rizos CV, Milionis HJ, Kostapanos MS, Florentin M, Kostara CE, Elisaf MS, Liberopoulos EN. Effects of rosuvastatin combined with olmesartan, irbesartan, or telmisartan on indices of glucose metabolism in Greek adults with impaired fasting glucose, hypertension, and mixed hyperlipidemia: a 24-week, randomized, openlabel, prospective study. Clin Ther. 2010;32(3):492-505.

15. Trinder P. Determination of glucose in blood using glucose oxidase with an alternative oxygen acceptor. Ann Clin Biochem. 1972;6:24-27

16. Thangavel P, Sumathiral K, Karthikeyan S, Ramaswamy M. Endocrine response of the freshwater teleost, Sarotherodon mossambicus (Peters) to dimecron exposure. Chemosphere. 2005;61(8):1083-1092.

17. Richmond W. Preparation and properties of a cholesterol oxidase from Nocardia sp. and its application to the enzymatic assay of total cholesterol in serum. Clin Chem. 1973;19(12):1350-1356.

18. Werner M, Gabrielson DG, Eastman J. Ultramicro determination of serum triglycerides by bioluminescent assay. Clin Chem. 1981;27(2):268-271.

19. Friedewald WT, Levy RI, Fredrickson DS. Estimation of the concentration of low-density lipoprotein cholesterol in plasma, without use of the preparative ultracentrifuge. Clin Chem. 1972;18(6):499-502.
20. Benson SC, Pershadsingh HA, Ho CI, Chittiboyina A, Desai P, Pravenec M, Qi N, et al. Identification of telmisartan as a unique angiotensin II receptor antagonist with selective PPARgamma-modulating activity. Hypertension. 2004;43(5):993-1002.

21. Ford ES, Giles WH, Dietz WH. Prevalence of the metabolic syndrome among US adults: findings from the third National Health and Nutrition Examination Survey. JAMA. 2002;287(3):356-359.

22. Choi JS, Song J. Effect of genistein on insulin resistance, renal lipid metabolism, and antioxidative activities in ovariectomized rats. Nutrition. 2009;25(6):676-685.

23. Weston-Green K, Huang XF, Deng C. Olanzapine treatment and metabolic dysfunction: a dose response study in female Sprague Dawley rats. Behav Brain Res. 2011;217(2):337-346.

24. Yamashita H, Yoda H, Maruki T, Toyoshima R. Telmisartan, an angiotensin II type-1 receptor blocker, improved insulin resistance in a schizophrenic patient treated with olanzapine: a case report. Prog Neuropsychopharmacol Biol Psychiatry. 2008;32(1):308-310.

25. Araki K, Masaki T, Katsuragi I, Tanaka K, Kakuma T, Yoshimatsu H. Telmisartan prevents obesity and increases the expression of uncoupling protein 1 in dietinduced obese mice. Hypertension. 2006;48(1):51-57.

26. Hamed GM, Bahgat NM, El-Agaty SM, Soliman GZ, Emara MM. Effects of a soybean protein diet on ovariectomised female albino rats subjected to myocardial infarction. Singapore Med J. 2010;51(10):781-789.

27. Coccurello R, Caprioli A, Conti R, Ghirardi O, Borsini F, Carminati P, Moles A. Olanzapine (LY170053, 2-methyl-4-(4-methyl-1-piperazinyl)-10H-thieno[2,3-b] $[1,5]$ benzodiazepine), but not the novel atypical antipsychotic ST2472 (9-piperazin-1-ylpyrrolo[2,1-b] [1,3]benzothiazepine), chronic administration induces weight gain, hyperphagia, and metabolic dysregulation in mice. J Pharmacol Exp Ther. 2008;326(3):905-911.

28. Del Valle MC, Loebel AD, Murray S, Yang R, Harrison DJ, Cuffel BJ. Change in framingham risk score in patients with schizophrenia: a post hoc analysis of a randomized, double-blind, 6-week trial of ziprasidone and olanzapine. Prim Care Companion J Clin Psychiatry. 2006;8(6):329-333.

29. Sofic E, Rustembegovic A, Pehlic E, Sapcanin A, Toromanovic J, Tahirovic I, Wichart I. Plasma lipids, lipoproteins and hormones levels during olanzapine treatment in psychosis and depression. Med Arh. 2007;61(2):71-72.

30. Sengupta P, Bagchi C, Sharma A, Majumdar G, Dutta C, Tripathi S. Olanzapine-induced hepatopathy in albino rats: A newer model for screening putative hepatoprotective agents, namely silymarin. Indian J Pharmacol. 2010;42(6):376-379.

31. Makita S, Abiko A, Naganuma Y, Moriai Y, Nakamura M. Effects of telmisartan on adiponectin levels and body 
weight in hypertensive patients with glucose intolerance. Metabolism. 2008;57(10):1473-1478.

32. Goyal BR, Mesariya P, Goyal RK, Mehta AA. Effect of telmisartan on cardiovascular complications associated with streptozotocin diabetic rats. Mol Cell Biochem. 2008;314(1-2):123-131.

33. Clemenz M, Frost N, Schupp M, Caron S, Foryst-Ludwig A, Bohm C, Hartge M, et al. Liver-specific peroxisome proliferator-activated receptor alpha target gene regulation by the angiotensin type 1 receptor blocker telmisartan. Diabetes. 2008;57(5):1405-1413.

34. Saito K, Ishizaka N, Hara M, Matsuzaki G, Sata M, Mori I, Ohno M, et al. Lipid accumulation and transforming growth factor-beta upregulation in the kidneys of rats administered angiotensin II. Hypertension. 2005;46(5):1180-1185.

35. Taslim S, Tai ES. The relevance of the metabolic syndrome. Ann Acad Med Singapore. 2009;38(1):29-25. 